
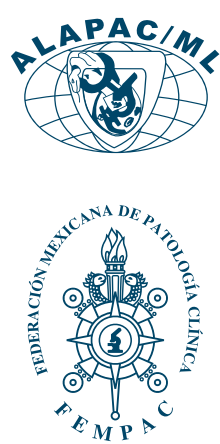

doi: $10.35366 / 103340$

\title{
Validación de un modelo para estimar la letalidad del COVID-19
}

\author{
Model validation to estimate the lethality of COVID-19
}

Simón Domínguez Jesús I,* Simón Domínguez Nadima, ${ }^{\ddagger}$ Reyes Núñez Miguel A ${ }^{\S}$

Palabras clave: Pandemia, COVID-19, letalidad.

Keywords: Pandemic, COVID-19, lethality.

* Director del Programa Integral de Ensayos de Aptitud de la Federación Mexicana de Patología Clínica. México. Programa Integral de Ensayos de Aptitud de la Federación Mexicana de Patología Clínica. México. ‡ Profesora Emérita, Facultad de Contaduría y Administración de la Universidad Nacional Autónoma de México. Miembro del Consejo de Honor de la Federación Mexicana de Universitarias. México. $\S$ Presidente de la Federación Mexicana de Patología Clínica. México.

Correspondencia: Jesús I Simón Domínguez Avenida 20 de Noviembre,

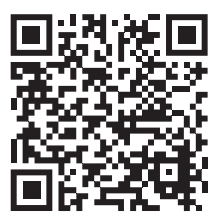

\section{RESUMEN}

\begin{abstract}
El presente documento tiene como objetivo dar a conocer los resultados para la validación del «Modelo COVID-19», el cual se publicó en junio de 2020, en el lapso del 1/1/2020 al 30/6/2021. Resultados: Aun cuando se desconoce el número total de casos infectados por el virus SARS-CoV-2, puede afirmarse que es mayor al número de casos confirmados conocidos, por lo cual en la letalidad calculada con los casos confirmados se obtiene un rango muy amplio 0.06-19.67\% (información del 30 de junio de 2021 de 147 países). Al término de 2020, se realizó una correlación con datos de Estados Unidos dado que fue el país con más pruebas diagnósticas realizadas en 2020 (257'732,886). En el segundo semestre realizaron 220 '366,235 pruebas, $85.5 \%$ del total realizadas en 2020 . Las diferencias entre los casos confirmados $\left(17^{\prime} 458,011\right)$ y los casos estimados del segundo semestre son muy pequeñas $(0.422 \%)$, la diferencia entre la letalidad fue de $0.005 \%$ y la diferencia de las prevalencias fue de $0.022 \%$. Este modelo es útil (con base en los resultados del segundo semestre de 2020) para estimar los casos infectados, la letalidad y la prevalencia. Se detallan otros datos con base en este modelo.
\end{abstract}

\section{ABSTRACT}

The purpose of this document is to present the results of the «Model COVID-19», in the period from 1/1/2020 to 6/30/2021, for its validation. This model was published in June 2020. Results: Even though the total number of cases infected by the SARS-CoV-2 virus is unknown, it can be affirmed that it is greater than the number of known confirmed cases. Therefore, the range obtained from the lethality calculated with confirmed cases is extremely wide: $0.06 \%$ to $19.67 \%$ (information obtained on June 30, 2021 from 147 countries). At the end of 2020, a correlation was made with data from the United States since it was the country with the highest number of diagnostic tests performed in 2020 (257'732,886). In the second semester, 220'366,235 tests were implemented, $85.5 \%$ of the total performed in 2020 . The difference between the confirmed cases $\left(17^{\prime} 458,011\right)$ and the cases estimated with our model for the second semester is minimal $(0.422 \%)$, additionally, the variation of lethality was $0.005 \%$ and the discrepancy on prevalence was $0.022 \%$. This model is useful to estimate infected cases, fatality and prevalence; based on the results of the second half of 2020. Other data is detailed based on this model.

\section{INTRODUCCIÓN}

-1 I objetivo del presente trabajo es reflexionar sobre la pandemia COVID-19, con el fin de encontrar los aspectos importantes de los que hasta este momento no se tienen evidencias concluyentes.

Después de realizar el análisis de la literatura académica, concluimos que no todas las preguntas se pueden responder en estos momentos o se requieren estudios específicos y de investigadores expertos.
Aun cuando se desconoce el número total de casos infectados por el virus SARS-CoV-2, puede afirmarse que es mayor al número de casos confirmados conocidos. Esto se debe principalmente a que las pruebas diagnósticas son limitadas y, por lo general, sólo se realizan en los casos sospechosos, por lo que el rango de la letalidad calculada con los casos confirmados es muy amplio, 0.06-19.67\% (información obtenida el 30 de junio de 2021 de 147 países). ${ }^{1}$

En junio de 2020, publicamos un modelo sencillo y empírico para poder estimar la letali-

Citar como: Simón DJI, Simón DN, Reyes NMA. Validación de un modelo para estimar la letalidad del COVID-19. Rev Mex Patol Clin Med Lab. 2021; 68 (2): 56-58. https://dx.doi.org/10.35366/103340 
Núm. 82, interior 205, Col. Centro, 06090, Alcaldía Cuauhtémoc Ciudad de México. Teléfono: 55 55221282.

E-mail: jsimon@ piensa.org.mx

Recibido: 16/09/2021 Aceptado: 28/09/2021 dad del COVID-19 y para responder la siguiente pregunta: ¿cuál es la cantidad de infectados por el SARS-CoV-2 en cada país? ${ }^{2}$ Asimismo, desarrollamos un programa abierto en Microsoft Excel ${ }^{\circledR}$, el cual nos permite obtener datos estimados de 147 países y que se actualiza cada día (http://letalidadcovid.cissmedical.com). Este modelo está basado en los factores de riesgo que influyen en una mayor mortalidad, el número de defunciones y una letalidad fija para cada país.

Por lo anterior, se presentan los resultados en el lapso del 1/1/2020 al 30/6/2021.

\section{RESULTADOS}

Al término de 2020, se realizó otra correlación con datos de Estados Unidos dado que fue el país con mayor número de pruebas diagnósticas realizadas en $2020\left(257^{\prime} 732,886\right)$, evaluando por semestre en forma independiente con los resultados siguientes:

1. Las diferencias entre el primero y segundo semestres son importantes dado que en el primero no se realizaron las pruebas diagnósticas requeridas debido a falta de éstas y sólo se aplicaron a pacientes sintomáticos graves o críticos (Figura 1).
2. Las diferencias entre los casos confirmados $\left(17^{\prime} 458,011\right)$ y los estimados del segundo semestre son muy pequeñas $(0.422 \%)$, entre la letalidad de confirmados y estimados fue de $0.005 \%$ y la diferencia de las prevalencias fue de $0.022 \%$. El número de casos confirmados en este semestre representa una muestra suficiente para validar el número de casos estimados (Tabla 1).

3. Las defunciones de un día no se relacionan con los nuevos casos de ese día, por lo que se deben correlacionar los datos totales obtenidos en un lapso de tiempo, mientras más grande es este lapso, mayor certidumbre tendrán las correlaciones.

4. Para poder calcular cuántas pruebas diagnósticas se requieren para obtener resultados óptimos $=(1 /$ letalidad $) /$ prevalencia*número de defunciones; si la letalidad y prevalencia se incrementan, las pruebas requeridas serán menos (Figura 2). Pruebas diagnósticas requeridas en el primer semestre $=(1 / 1.281 \%) / 3.01 \%=$ $78.1 / 0.0301=2,565 *$ número de defunciones $=2,595 * 127,471=330^{\prime} 747,013$ pruebas requeridas; pruebas realizadas: 37'366,651 (11.30\% de las requeridas). Pruebas diagnósticas requeridas en el segundo semestre $=(1 / 1.281 \%) / 8.3 \%=78.1 / 0.083$

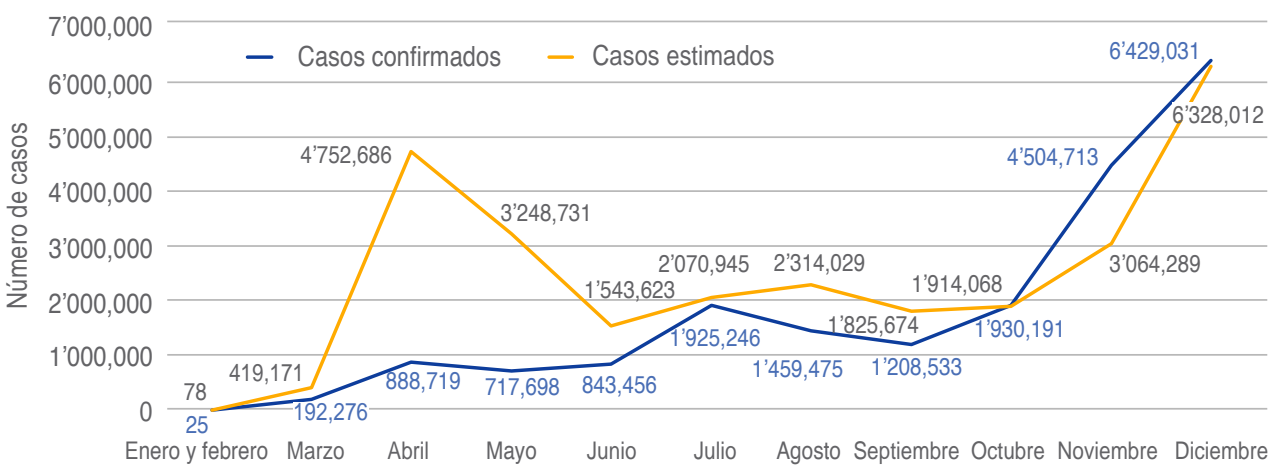

Figura 1:

Casos confirmados y estimados en Estados Unidos 2020. Modificada de: Roser $\mathrm{M}$ et al.

Tabla 1: Validación de casos estimados.

\begin{tabular}{|c|c|c|c|c|c|}
\hline Estados Unidos & Casos confirmados & Casos estimados & Diferencias & Defunciones & Población \\
\hline Total de 01/07/2020 a 31/12/2020 & $17^{\prime} 458,011$ & $17^{\prime} 531,620$ & $0.422 \%$ & 224,514 & $331^{\prime} 002,647$ \\
\hline Letalidad (\%) & 1.286 & 1.281 & 0.005 & & \\
\hline Prevalencia (\%) & 5.274 & 5.297 & 0.022 & & \\
\hline
\end{tabular}




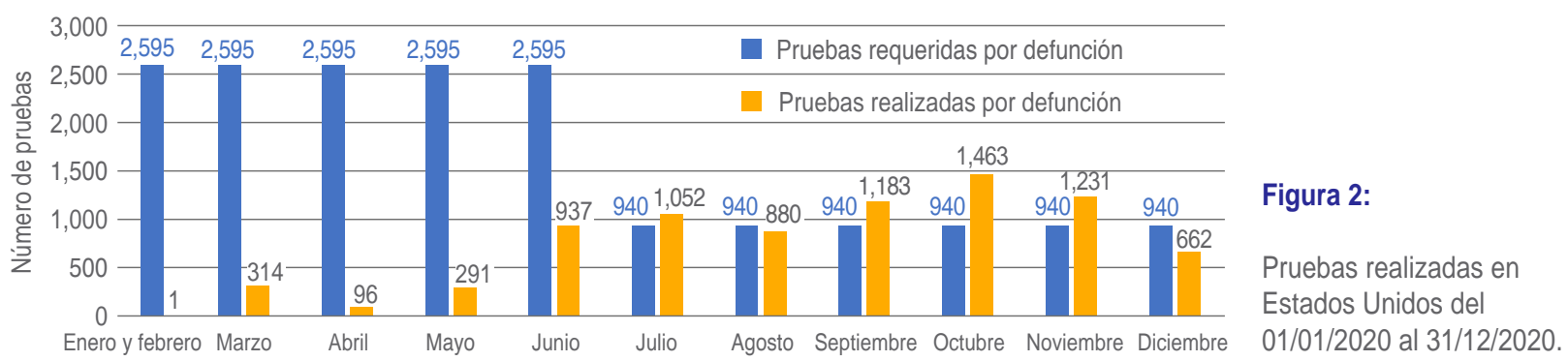

$=940 *$ número de defunciones $=940 * 224,527=$ $211^{\prime} 055,380$ pruebas requeridas; pruebas realizadas: $220^{\prime} 366,235$ (4.41\% más de las requeridas).

5. En el primer semestre de 2021, se observa un incremento de defunciones de $11.1 \%$, los casos confirmados disminuyeron $22.3 \%$ con respecto al segundo semestre de 2020 y $31.22 \%$ respecto a los casos estimados, las pruebas diagnósticas disminuyeron en $3.4 \%$. Dado que el número de casos confirmados disminuyó y las defunciones se incrementaron, existe incertidumbre respecto a la causa, si es por falta de pruebas diagnósticas, por efectos de las vacunas o por las mutaciones del SARS-CoV-2. Para resolver lo anterior, se requieren datos adicionales como las edades de las defunciones, cuántas defunciones se infectaron después de la aplicación de la vacuna y las mutaciones del SARS-CoV-2 en los pacientes que fallecieron.

\section{CONCLUSIONES}

Este modelo es útil (con base en los resultados del segundo semestre de 2020) para estimar la letalidad, la prevalencia y sus variaciones de un país a otro (se usa la misma vara de medición), lo cual es esencial para apoyar a los gobiernos en la elección de estrategias apropiadas y no causar incertidumbre en la población.
Se requieren más datos para poder sustentar los cambios en el primer semestre de 2021 como el número de defunciones con vacunación previa, el tipo de vacunas utilizadas, las mutaciones del SARS-CoV-2, las edades y las comorbilidades de las defunciones.

$\mathrm{Si}$ bien las hospitalizaciones son importantes para disminuir la mortalidad, no influyen en la cantidad de casos infectados.

Las estimaciones realizadas pueden ser diferentes a la realidad, pero los datos reales sólo podrán conocerse hasta tener los resultados de los estudios de seroprevalencia (presencia de anticuerpos $\lg M$ e $\operatorname{lgG}$ ), los cuales se llevan a cabo al término de los brotes; si tomamos en cuenta que en la actualidad se está aplicando un número importante de vacunas que interfieren en estos estudios de seroprevalencia, se deberán realizar pruebas específicas para este fin, este modelo podrá utilizarse para estimar la prevalencia al término de la pandemia.

\section{REFERENCIAS}

1. Roser M, Ritchie H, Ortiz-Ospina E, Hasell J. Coronavirus pandemic (COVID-19). Published online at OurWorldInData.org. 2020. Available in: https://ourworldindata.org/coronavirus

2. Simón DJI, Simón DN, Reyes NMA. Cómo estimar la letalidad del COVID-19. Rev Mex Patol Clin Med Lab. 2020; 67 (1): 4-8. doi: 10.35366/93845. 